\title{
Application of an Integrative Computational Framework in Trancriptomic Data of Atherosclerotic Mice Suggests Numerous Molecular Players
}

\author{
Olga Papadodima, ${ }^{1}$ Allan Sirsjö, ${ }^{2}$ Fragiskos N. Kolisis, ${ }^{3}$ and Aristotelis Chatziioannou ${ }^{1}$ \\ ${ }^{1}$ Metabolic Engineering and Bioinformatics Program, Institute of Biological Research and Biotechnology, National Hellenic Research \\ Foundation, 48 Vas. Constantinou Avenue, 11635 Athens, Greece \\ ${ }^{2}$ Division of Clinical Medicine, School of Health and Medical Sciences, Örebro University, Örebro SE-701 82, Sweden \\ ${ }^{3}$ Biotechnology Laboratory, School of Chemical Engineering, Zografou Campus, National Technical University of Athens, \\ 15780 Athens, Greece
}

Correspondence should be addressed to Allan Sirsjö, allan.sirsjo@oru.se and Aristotelis Chatziioannou, achatzi@eie.gr

Received 25 May 2012; Accepted 21 September 2012

Academic Editor: Konstantina Nikita

Copyright ( $) 2012$ Olga Papadodima et al. This is an open access article distributed under the Creative Commons Attribution License, which permits unrestricted use, distribution, and reproduction in any medium, provided the original work is properly cited.

\begin{abstract}
Atherosclerosis is a multifactorial disease involving a lot of genes and proteins recruited throughout its manifestation. The present study aims to exploit bioinformatic tools in order to analyze microarray data of atherosclerotic aortic lesions of ApoE knockout mice, a model widely used in atherosclerosis research. In particular, a dynamic analysis was performed among young and aged animals, resulting in a list of 852 significantly altered genes. Pathway analysis indicated alterations in critical cellular processes related to cell communication and signal transduction, immune response, lipid transport, and metabolism. Cluster analysis partitioned the significantly differentiated genes in three major clusters of similar expression profile. Promoter analysis applied to functional related groups of the same cluster revealed shared putative cis-elements potentially contributing to a common regulatory mechanism. Finally, by reverse engineering the functional relevance of differentially expressed genes with specific cellular pathways, putative genes acting as hubs, were identified, linking functionally disparate cellular processes in the context of traditional molecular description.
\end{abstract}

\section{Introduction}

Atherosclerosis is the leading pathological contributor to cardiovascular morbidity and mortality worldwide, characterized by the progressive accumulation of lipid and fibrous depositions in the vessel wall of medium-sized and large arteries. Although it has traditionally been viewed as simple deposition of lipids within the vessel wall, it is now assumed that atherosclerosis is a multifactorial disease that involves several genes and proteins, activated during its genesis, progress, and phenotypic manifestation. During atherogenesis, a complex endothelial activation and dysfunction induced by elevated and modified low-density lipoproteins and many other factors leads to a compensatory inflammatory response [1]. Current evidence supports a central role for inflammation, in all phases of the atherosclerotic process. Substantial biological data implicate inflammatory pathways in early atherogenesis, in the progression of lesions, and finally in the thrombotic complications of this disease [2].

Clinical investigations, population studies, and cell culture experiments have provided important clues to the pathogenesis of atherosclerosis. However, the use of animal models has had a crucial contribution in the research of the atherosclerotic course. Atherosclerosis will not be developed in laboratory mice under normal conditions. However, targeted deletion of the gene for Apolipoprotein $\mathrm{E}$ (ApoE knockout mice) leads to severe hypercholesterolemia 


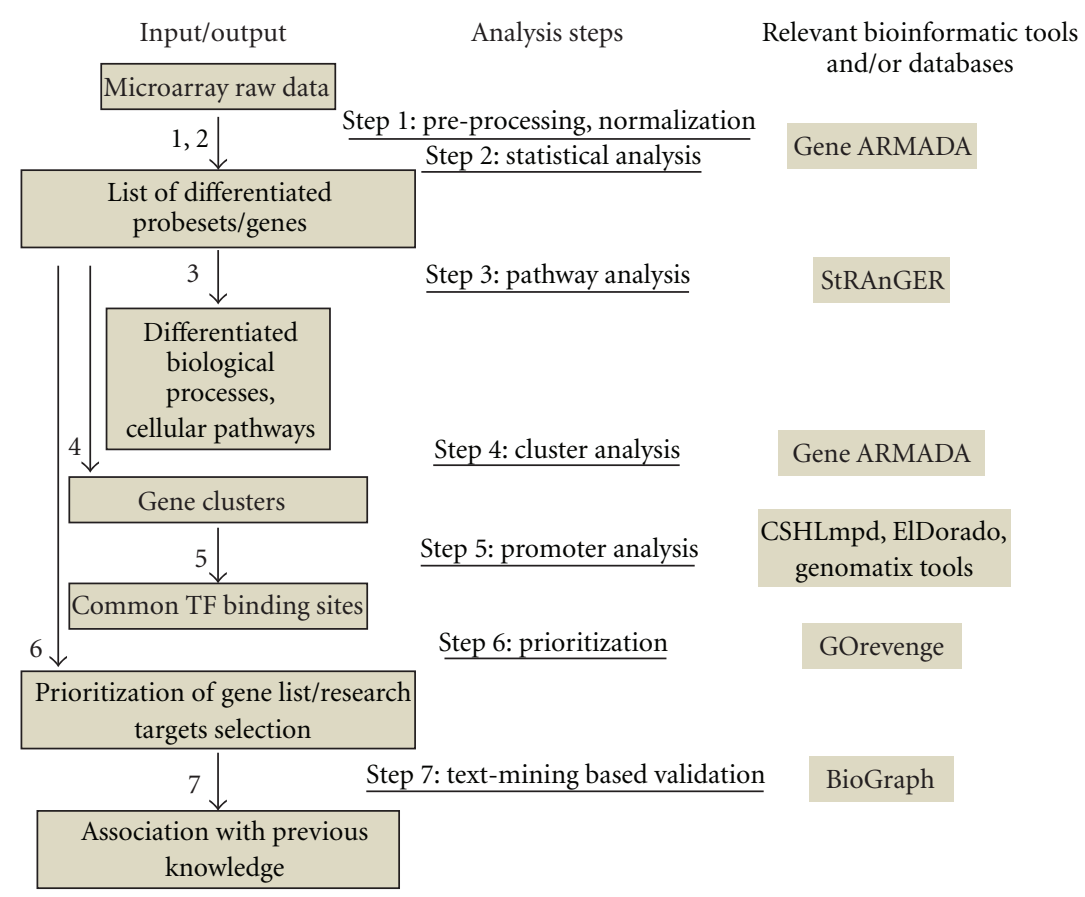

FIGURE 1: Schematic representation of the proposed workflow. Arrows depict the various analysis steps. The bioinformatic tools and databases used for the implementation of each step are also shown.

and spontaneous atherosclerosis [3]. For this reason, ApoE deficient mice are widely used to study atherosclerosis [4]. ApoE is a ligand for receptors that clear chylomicrons and very low-density lipoprotein remnants. Furthermore, a number of population studies suggest that ApoE genotype predicts the risk of developing atherosclerosis and related diseases [5].

In this study, we propose a framework for efficient translational bioinformatic analysis showcased on a microarray dataset concerning biological specimen from ApoE knockout mice. Gene expression data of wild type and ApoE knockout 6-, 32-, and 78-week-old mice have been utilized. This dataset was previously presented in a detailed work studying atherosclerosis and inflammatory pathways during aging [6]. The proposed workflow comprises seven basic steps (Figure 1): raw data pre-processing and normalization, statistical selection, pathway analysis, clustering, promoter analysis, gene list prioritization, exploiting network centrality criteria enabling identification of interesting research targets, and finally intelligent text-mining based validation of the selected molecular targets from the broader biomedical literature. Aim here is to combine several bioinformatic tools, in a unique, generic, computational workflow, appropriate for batch processing, able to confer reliable functional knowledge, regarding different aspects of the biological mechanism investigated, in order to highlight critical, underlying, molecular determinants governing it. Besides an algorithmic proposition, the workflow presented here is currently in the phase of implementation, regarding the seamless integration of its constituent modules, exploiting the web service technology [6], accessible through a userfriendly web application, and enabling automated extraction of consolidated biological knowledge, in the form of concrete functional scenario, from high-volume data omic datasets. Nowadays, several tools are available for the implementation of each analysis step. For instance, Bioconductor [7] represents one of the richest repositories of statistical algorithms and has become, by all means, a standard for microarray data analysis but its command line interface limits its usability to many, wet-lab oriented, biological experts. To overcome this limitation, user friendly software packages for normalization, statistical analysis, and visualization of microarray expression data have been developed, like Gene ARMADA [8] and FlexArray [9]. Regarding pathway analysis, multiple software tools are exploiting ontological vocabularies to target the issue of detecting over-represented terms in microarray datasets, aiming to indicate possibly altered molecular processes [10-14]. Regarding promoter analysis, it remains one of the most intricate issues regarding the efficient mining of gene lists derived from transcriptomic experiments. Different promoter sequence databases $[15,16]$ and bioinformatic tools $[17,18]$ have been developed but still elucidation of gene transcription regulating networks remains a great challenge. The derivation of functional information regarding gene function, exploiting semantic similarity criteria, represents a promising, yet fuzzy and increasingly bewildering in its interpretation, approach. Several criteria and measures have been proposed [19], however it is GOrevenge [20], which instead of focusing in the neighboring genes, it highlights linker genes, associated 
with discrete cellular functions (distant in terms of semantic similarity). Finally BioGraph [21] is a data integration and data mining platform for the exploration and discovery of biomedical information. The platform offers prioritizations of putative disease genes, supported by functional hypotheses. BioGraph can retrospectively confirm recently discovered disease genes and identify potential susceptibility genes, without requiring prior domain knowledge, outperforming from other text-mining applications in the field of biomedicine. In the present analysis we show that integration of different analysis snapshots, as obtained through bioinformatic analyses, results in reliable, prioritized and informative lists of differentiated genes, and/or molecular pathways.

\section{Materials and Methods}

2.1. Microarray Data. The mouse dataset used is the GSE 10000, available at Gene Expression Omnibus (GEO) database. Microarrays were prepared following MIAME guidelines, as described in [22]. Briefly, RNA from aortic tissue of ApoE knockout and wild type animals was hybridized on Affymetrix 4302.0 Arrays. Three different ages were studied: 6,32 , and 78 weeks.

2.2. Microarray Data Analysis and Statistical Analysis. Microarray data analysis was performed in Gene ARMADA [8]. Briefly, background correction was performed employing its gcRMA method followed by Quantile Normalization. Data were $\log 2$ transformed to comply with the normality assumption. Differentially expressed genes in at least one among all the experimental conditions were identified using Gene ARMADA, by performing 1-way ANOVA on $\log 2$ transformed fold changes. The resulting gene list was obtained by setting the $P$ value threshold to 0.01 , the False Discovery Rate (FDR) threshold to 0.05 and by removing genes that presented a fold change below $|1|$, in $\log 2$ scale, in all conditions.

2.3. Prioritized Pathway/Functional Analysis. Statistical enrichment analysis was performed using StRAnGER [8], in order to highlight biological processes including statistically significant numbers of the ANOVA derived genes. In order to expand our knowledge regarding the functional implication of genes in various cellular processes, prioritizing them according to their centrality, we used the online tool GOrevenge [20] with the following settings: Aspect: BP (Biological Process), Distance: Resnik, Algorithm: BubbleGene, and Relaxation: 0.15.

2.4. Cluster and Promoter Analysis. The list derived from ANOVA was subjected to hierarchical clustering (linkage method: Average, distance: Cosine) in Gene ARMADA. Promoter sequences from -700 to +300 , relative to transcription start site, were downloaded for mouse and human from Cold Spring Harbor Laboratory Mammalian Promoter Database (CSHLmpd) [16]. In the cases that alternative promoters were given for the same gene, we selected the one defined as the "best" at [16]. For promoters that we could not detect in this database, we additionally searched the ElDorado database [15]. In the case of genes with multiple promoters supported by different transcripts, we selected the one corresponding to the Reference Sequence of NCBI. To analyze each promoter set for common TF binding sites, we used the MatInspector software [18]. The parameters used were as follows: Library version: Matrix Library 8.0, Matrix group: Vertebrates, Transcription Factor sites common to: $85 \%$ of input sequences, Core similarity: 0.75 , Matrix similarity: Optimized, and $P$ value cut-off was set at 0.01 . Among the identified TF sites only those that were present in both species were considered.

\section{Results}

3.1. Statistically Significant Differentiated Genes. To obtain the aortic gene expression profile of ApoE deficient mice in 6-, 32-, and 78-week-old mice we analyzed the GSE 10000 dataset, containing expression data of aortic tissue from wild type and ApoE knockout mice. Specifically, in order to identify significant alterations among all three tested ages, 1way ANOVA was applied to expression fold changes between expression in ApoE knockout and wild type animals $(P$ value $<0.01$ and FDR $<0.05)$ coupled with further filtering on fold change $(>|1|$ in at least one condition in $\log 2$ scale). A list of 1033 significantly differentiated probesets was obtained (Supplementary Table 1; see supplementary material available online at doi:10.1155/2012/453513), depicted per time point using a volcano plot representation (Figure 2). These 1033 probesets correspond to 852 annotated genes. It is characteristic that in 6 weeks old mice the number of significantly altered genes is very limited, in 32 weeks old mice the majority of differentiated genes are upregulated, while in 78 weeks old mice we have the greater number of differentiated genes.

3.2. Pathway Analysis. For the scope of gaining further insight concerning the biological functionalities of gene expression alterations in a more systematic way, the list of 852 significantly differentiated genes yielded from ANOVA was subjected to statistical enrichment analysis using StRAnGER, exploiting GO terms and Kegg pathways for the task of the functional annotation of the interrogated genes. GO-based analysis, focused on the categories of "Biological Process" with a hypergeometric $P$ value $<0.001$, suggested several processes as possibly differentiated, which are presented in Table 1. A lot of central molecular mechanisms emerge as altered, as indicated by the GO categories listed in Table 1, like differentiation, proliferation (inferred by cell cycle and cell division GO terms), apoptosis, cell adhesion, signal transduction, and immune response. Kegg pathway-based analysis also indicates alterations in cytokine signaling, cell adhesion, and signal transduction (Supplementary Table 2 ). It is important to note that in conformity to the well established relationship of atherosclerosis and inflammation, the majority (29 out of 32 ) of the genes under the category "immune response" are upregulated suggesting a stimulation of the immunological mechanisms (Table 2). 


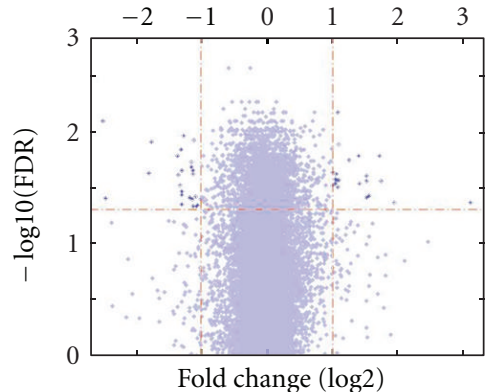

(a)

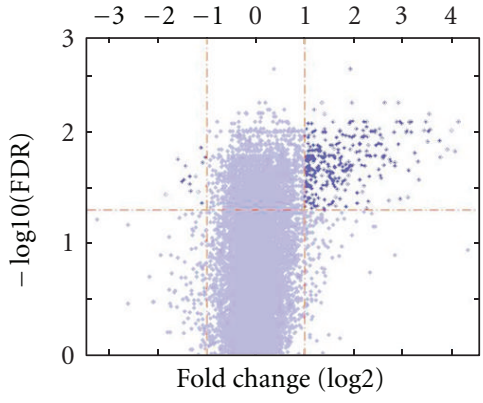

(b)

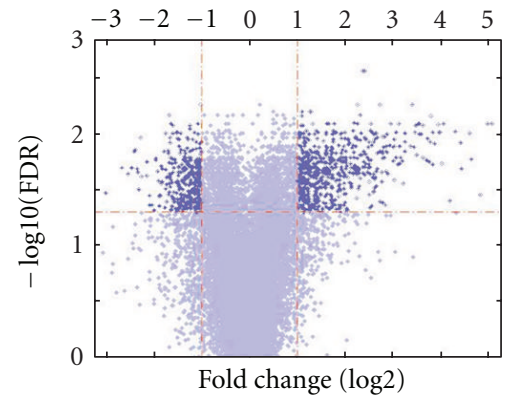

(c)

Figure 2: Volcano plots of the gene list as yielded by ANOVA. Each panel represents filtered and normalized data from each experimental condition (3, 6, and 78 weeks old mice). The horizontal axes depict the fold change ratio between ApoE deficient and wild type mice, for each age in $\log 2$ scale, while the vertical axes represent statistical significance by depicting the $-\log 10$ (FDR).

TABLE 1: GO-analysis. The list of 852 significantly altered genes was submitted to GO analysis elucidating over-represented GO terms. GOT $P$ value represents the hypergeometric test $P$ value score for each GO term. Enrichment represents the ratio of the number of times a GO term occurs in the 852 gene list to the number of times this GO term exists in the list of the Affymetrix 4302.0 array.

\begin{tabular}{lcc}
\hline GO annotation & GOT $P$ value & Enrichment \\
\hline Ion transport & 0.00000000003 & $33 / 498$ \\
Signal transduction & 0.00000000004 & $44 / 803$ \\
Cell differentiation & 0.00000000005 & $36 / 480$ \\
Immune response & 0.00000000005 & $32 / 250$ \\
Metabolic process & 0.00000000007 & $38 / 542$ \\
Cell adhesion & 0.00000000011 & $36 / 387$ \\
Protein amino acid & 0.00000000059 & $32 / 497$ \\
phosphorylation & & \\
Multicellular organismal & 0.00000000128 & $41 / 770$ \\
development & & \\
Proteolysis & 0.00000000690 & $25 / 358$ \\
Apoptosis & 0.00000010814 & $24 / 383$ \\
Lipid metabolic process & 0.00000328813 & $15 / 212$ \\
Protein transport & 0.00003352383 & $22 / 465$ \\
G-protein coupled receptor & 0.00028681297 & $19 / 436$ \\
signaling & 0.00034119987 & $21 / 510$ \\
Oxidation reduction & 0.00043684087 & $18 / 417$ \\
Cell cycle & 0.00050194998 & $12 / 231$ \\
Cell division & & \\
\hline
\end{tabular}

3.3. Cluster Analysis. In order to identify groups of genes presenting similar expression and possibly comprising regulated "waves" of transcription, the list of 1033 significantly differentiated probesets was subjected to hierarchical clustering (Figure 3). Three major clusters can be distinguished: the first one (323 probesets) contains transcripts downregulated in 78 week old mice, while their expression remains close to the control (wild type) level at 6 and 32 weeks. The second cluster (526 probesets) groups genes which are upregulated at 32 weeks and their expression at ApoE knockout mice remains at high levels, as compared to wild type, also at 78

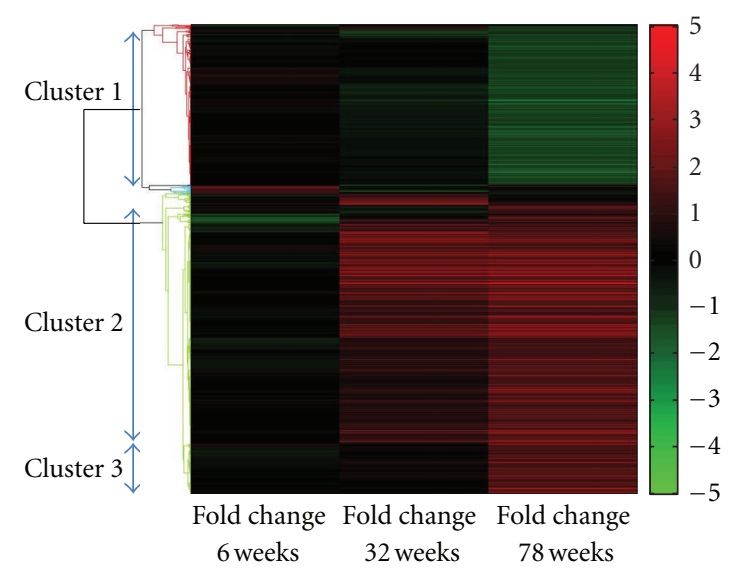

FIGURE 3: Hierarchical clustering of the 1033 statistically significant differentiated probesets. Fold changes between the gene expressions in ApoE knockout as compared to age-matched wild type mice are grouped in three major clusters.

weeks. The third cluster (110 probesets) groups genes whose expression is late upregulated at 78 week old ApoE knockout, as compared to age-matched wild type mice.

Based on these three major clusters, we performed GOanalysis to the genes of each cluster separately. Genes under cluster 1 are functionally connected to processes involved in cell differentiation, adhesion, and signal transduction. Cluster 2 contains the greatest number of genes, which are related mainly to mechanisms involved in immune and inflammatory response as well as lipid metabolism. These processes emerge as significantly altered specifically in the case of cluster 2. Cluster 2 genes are also connected to key cellular processes like signal transduction, apoptosis, cell cycle, and differentiation. Cluster 3 genes are mainly related to mechanisms concerning gene transcription.

3.4. Promoter Analysis. Next, we focused our analysis on small groups of genes presenting similar expression profile, as indicated by cluster analysis, and also being functional relevant, as suggested by $\mathrm{GO}$ analysis. In order to investigate 
TABle 2: Expression of genes under the GO term immune response. Values in the three last columns depict fold changes between ApoE knockout and age-matched wild type mice in $\log 2$ scale. The majority of genes at 32 and 78 weeks are upregulated.

\begin{tabular}{|c|c|c|c|c|}
\hline Symbol & Description & 6 weeks & 32 weeks & 78 weeks \\
\hline Ccl6 & Chemokine (C-C motif) ligand 6 & -0.17 & 2.25 & 2.23 \\
\hline $\mathrm{Cd} 74$ & $\begin{array}{l}\text { CD74 antigen (invariant polypeptide of major histocompatibility complex, class II } \\
\text { antigen-associated) }\end{array}$ & 0.29 & 1.56 & 1.43 \\
\hline $\mathrm{Clqb}$ & Complement component 1 , q subcomponent, beta polypeptide & -0.23 & 2.28 & 2.43 \\
\hline $\mathrm{H} 2-\mathrm{Ab} 1$ & Histocompatibility 2, class II antigen A, beta 1 & -0.25 & 1.94 & 2.77 \\
\hline C1qa & Complement component 1 , q subcomponent, a polypeptide & -0.08 & 1.98 & 2.18 \\
\hline Tlr2 & Toll-like receptor 2 & 0.14 & 0.99 & 1.38 \\
\hline $\mathrm{Ccl} 7$ & Chemokine (C-C motif) ligand 7 & -0.98 & 1.59 & 4.42 \\
\hline $\mathrm{C} 4 \mathrm{~b}$ & Complement component 4B (Childo blood group) & 0.23 & 1.2 & 1.74 \\
\hline Cblb & Casitas B-lineage lymphoma b & 0.2 & 0.34 & 1.36 \\
\hline Fcgr $2 b$ & Fc receptor, IgG, low affinity IIb & -0.1 & 1.93 & 2.19 \\
\hline $\mathrm{Cd} 300 \mathrm{lb}$ & CD300 antigen like family member B & -0.46 & 2.83 & 2.87 \\
\hline Susd2 & Sushi domain containing 2 & 0.22 & -0.08 & -1.07 \\
\hline $\mathrm{Ccl} 8$ & Chemokine (C-C motif) ligand 8 & 0.33 & 2.92 & 3.01 \\
\hline $\mathrm{Cd} 14$ & CD14 antigen & -0.14 & 1.52 & 2.44 \\
\hline Fcgr1 & Fc receptor, IgG, high affinity I & -0.05 & 1.82 & 2.03 \\
\hline Cadm1 & Cell adhesion molecule 1 & -0.57 & 1.5 & 2.01 \\
\hline $\mathrm{C} 2$ & Complement component 2 (within $\mathrm{H}-2 \mathrm{~S}$ ) & -0.81 & 1.02 & -0.03 \\
\hline Clec7a & C-type lectin domain family 7 , member a & 0.15 & 3.52 & 3.86 \\
\hline Procr & Protein C receptor, endothelial & -0.25 & 0.77 & 1.02 \\
\hline Clqc & Complement component $1, \mathrm{q}$ subcomponent, $\mathrm{C}$ chain & -0.22 & 2.22 & 2.25 \\
\hline Ccl19 & Chemokine (C-C motif) ligand 19 & -0.71 & 1.07 & 1.64 \\
\hline Enpp3 & Ectonucleotide pyrophosphatase/phosphodiesterase 3 & 0.24 & -0.00 & -1.28 \\
\hline $\mathrm{Cx} 3 \mathrm{cll}$ & Chemokine (C-X3-C motif) ligand 1 & 0.18 & 1.71 & 1.87 \\
\hline Ccl9 & Chemokine (C-C motif) ligand 9 & -0.46 & 1.74 & 2.19 \\
\hline $\mathrm{H} 2-\mathrm{Eb} 1$ & Histocompatibility 2, class II antigen E beta & 0.19 & 1.62 & 2.17 \\
\hline $\mathrm{H} 2-\mathrm{Aa}$ & Histocompatibility 2 , class II antigen A, alpha & 0.95 & 2.39 & 2.34 \\
\hline Cxcl12 & Chemokine (C-X-C motif) ligand 12 & -0.34 & 1.1 & 1.95 \\
\hline Enpp1 & Ectonucleotide pyrophosphatase/phosphodiesterase 1 & 0.14 & 0.37 & 1.08 \\
\hline Rnf19b & Ring finger protein $19 \mathrm{~B}$ & -0.29 & 0.73 & 2.58 \\
\hline $\operatorname{Prg} 4$ & Proteoglycan 4 & 0.16 & 3.33 & 3.99 \\
\hline Irf8 & Interferon regulatory factor 8 & 0.28 & 1.9 & 2.61 \\
\hline Cxcl1 & Chemokine (C-X-C motif) ligand 1 & -0.46 & 1.77 & 2.66 \\
\hline
\end{tabular}

whether there are common regulatory transcriptional mechanisms in such groups of genes, we performed a representative promoter analysis in genes of cluster 2 belonging to the GO category of "immune response" either "inflammatory response." We selected these categories because they appear as significantly altered, scoring at the top of GO analysis prioritization list. We combined the genes of these two groups, resulting at a total number of 36 genes in both categories, because they are functionally relevant, as they represent genes involved in immunological mechanisms. In order to find common putative transcription factor (TF) binding sites in at least a subset of this group, proximal promoter sequences from both mouse and human genomes were extracted from available databases and analyzed as described in Methods. Only common TF binding sites among the two species were considered. Table 3 summarizes statistically significant TF motif families common in at least $80 \%$ of promoter sequences, sorted in descending order in terms of statistical significance. The $P$ values, representing the probability to obtain a greater or equal number of sequences with a match in a random sample of the same size as the input sequence set, are precalculated for each binding site and depend on its definition.

We then examined whether among the significantly differentiated genes we could identify TFs possibly recognizing binding sites presented at Table 3 and thus being involved in the regulation of the relevant genes. Interestingly, among the upregulated genes there are Klf4 and Irf8 TFs, whose binding sites are found at $100 \%$ and $86 \%$ of the tested promoters, respectively. In particular, Klf4 shows an increased expression 
TAвLe 3: Common TF motif families in the promoters of 36 genes belonging to cluster 2 and to the categories "immune response" and "inflammatory response." The percentage column depicts the percentage of genes whose promoters have at least one match with the respective motif family. Percentages and $P$ value calculations are based on mouse promoters.

\begin{tabular}{|c|c|c|c|}
\hline Family & Description & $P$ value & $\%$ \\
\hline V\$CTCF & CTCF and BORIS gene family & 0.00000185 & 86 \\
\hline V\$MZF1 & Myeloid zinc finger 1 factors & 0.00000878 & 89 \\
\hline V\$EGRF & EGR/nerve growth factor induced protein $\mathrm{C}$ and related factors & 0.00011981 & 86 \\
\hline V\$SRFF & Serum response element binding factor & 0.00012387 & 83 \\
\hline V\$PLAG & Pleomorphic adenoma gene & 0.00015650 & 86 \\
\hline V\$GREF & Glucocorticoid responsive elements & 0.00016410 & 92 \\
\hline V\$KLFS & Krueppel like transcription factors & 0.00024104 & 100 \\
\hline V\$GLIF & GLI zinc finger family & 0.00170920 & 81 \\
\hline V\$STAT & Signal transducer and activator of transcription & 0.00177177 & 92 \\
\hline V\$PAX5 & PAX-2/5/8 binding sites & 0.00246076 & 89 \\
\hline $\mathrm{V} \$ \mathrm{E} 2 \mathrm{FF}$ & E2F-myc activator/cell cycle regulator & 0.00400401 & 86 \\
\hline $\mathrm{V} \$ \mathrm{XBBF}$ & X-box binding factors & 0.00470139 & 89 \\
\hline V\$GATA & GATA binding factors & 0.00499105 & 94 \\
\hline V\$PAX6 & PAX-4/PAX-6 paired domain binding sites & 0.00504198 & 89 \\
\hline V\$ETSF & Human and murine ETS1 factors & 0.00646776 & 100 \\
\hline V\$GCMF & Chorion-specific TFs with a GCM DNA binding domain & 0.00921126 & 83 \\
\hline V\$HEAT & Heat shock factors & 0.01639280 & 92 \\
\hline V\$RXRF & RXR heterodimer binding sites & 0.01834270 & 97 \\
\hline V\$FKHD & Fork head domain factors & 0.02095320 & 94 \\
\hline V\$IRFF & Interferon regulatory factors & 0.02221250 & 86 \\
\hline V\$HAND & Twist subfamily of class B bHLH transcription factors & 0.03199970 & 94 \\
\hline $\mathrm{V} \$ \mathrm{ABDB}$ & Abdominal-B type homeodomain transcription factors & 0.04170260 & 89 \\
\hline
\end{tabular}

at ApoE knockout mice as compared to age-matched wild type both at 36 and 78 weeks ( 0.62 and 1 fold increase, in $\log 2$ scale, resp.) while in 6 weeks the expression of KLf4 is moderately decreased as compared to wild type $(-0.46$, $\log 2$ scale). The expression of Irf8 at ApoE knockout mice is significantly increased both at 36 and 78 weeks (1.9 and 2.61 fold increase, in $\log 2$ scale, resp.), while at 6 weeks it remains at the wild type levels. Thus the upregulation of these factors could partially account for the observed upregulation of the immune-related group of genes.

3.5. Identification of Candidate Hub-Genes. In order to expand our knowledge regarding which genes have critical role, taking into consideration their centrality as described in the GO tree, we used the online tool GOrevenge [20]. The list of 852 differentiated genes was submitted to GOrevenge and the analysis was performed based on GO annotations for Mus musculus as described in materials and methods section. The derived list of genes, containing candidate linker genes, that is genes participating in many different cellular processes, was partitioned to include only the genes that have been also identified, as statistically significantly differentiated. The derived list (Table 4) contains genes that were identified as significant both by ANOVA and by GOrevenge analysis. The list of genes is prioritized according to the centrality of each gene, as it is reflected by the number of GO biological processes related terms remaining after GOrevenge pruning [20]. Significant molecules involved in signaling and developmental mechanisms emerge as central players. In order to evaluate the relation of these genes with atherosclerosis, which is a principle phenotypic characteristic of ApoE knockout mice, we used the BioGraph platform [21] which utilizes data mining algorithms that exploit textual terms to build a network of heterogeneous relations which link genes with a specific concept (such as genes, proteins, diseases as described in [21]). The resultant BioGraph network describes associations in Homo sapiens. By setting atherosclerosis as concept, the relation of each gene with atherosclerosis was assessed and the top 20 genes obtained by GOrevenge were prioritized as shown in Table 5 by BioGraph algorithm. The genes are prioritized according to their score which is a statistical enrichment measure of the relevance of each gene with the inquired context (here specified as atherosclerosis) to the total relations (references) of the gene in the universe of terms. In this way, the user can derive which of its genes are already associated and in what extent with a given disease or generally biological term, and which of them represent novel findings with respect to the investigated pathological phenotype. Since the list of gene symbols used as input to BioGraph represent Mus musculus genes, while BioGraph refers to Homo sapiens genes, some of them could have different symbol in each species. In the case that a gene symbol was not recognized by BioGraph, we searched 
the NCBI HomoloGene database [23] in order to find the homologous gene in Homo sapiens (e.g., FOXF1 of Table 5 corresponds to Foxfla of Table 4).

\section{Discussion}

In this study, we presented a detailed, multi-stage, translational bioinformatic analysis of ApoE knockout mice, exploiting different methods in order to identify critical altered molecular mechanisms and important central players. Our approach was to apply a generic computational framework, which exploits rigorous statistical or computational measures at every analytical step, for the efficient systems level interpretation of the results of ApoE dataset. The workflow proposed here integrates various software products, in a unified translational pipeline, able to cope with versatile, high-volume investigation tasks, and at the same time provide a reliable systemic interpretation for the biological mechanism studied. In this way, a powerful translational backbone is set, which connects the wetlab part with the theoretical knowledge for the biological problem interrogated, as rescued in molecular databases, controlled ontological vocabularies or the literature. The workflow presented in this study, currently in the phase of implementation as regards to its software components integration, represents an efficient and highly innovative effort, either in terms of speed of analytical performance, as well as real biological value of the results. This is so because it provides results which are qualified from a composite framework that combines ideally both individual and group quality measures, together with an insightful comprehension of the underlying topological networks, actively involved in the mechanism studied. The correlation of the results of the molecular analysis with literature-derived associations manages to highlight and propose promising, novel candidates that have not been studied in the context of the given pathology. They could thus represent ideal targets for further biological experimentation. Maximizing the total information gain encompassing all analytical steps of the proposed workflow represents a critical parameter regarding the implementation of the web application. However as the derivation of automated statistical thresholds for such highvolume data processing in an unsupervised manner both in terms of performance and computational speed is a very challenging task, this still remains an open issue for extensive research work and testing, representing an important point for future work.

Computational technologies are complementary to conventional "wet lab" gene discovery technologies in that they can support the prioritization and comprehension of high-volume molecular data (i.e., omic datasets from a microarray or novel sequencing technologies, associated regions from genome wide association or linkage studies) enabling the efficient selection of the top candidates, under a range of criteria, for further study. In recent years, there are popular tools and environments in the scientific computing realm (data-mining, artificial intelligence, hypercomputing), like the Taverna workflow manager [24] or the RapidMiner solution, formerly known as YALE [25], which enable efficient workflow integration and deployment, exploiting versatile web service repositories, containing hundreds of operators implementing various analytical tasks. Especially Taverna workflow manager, through myExperiment (http://www.myexperiment.org) [26] and Biocatalogue (http://www.biocatalogue.org) [27], integrates an impressive number of workflows and web services all accessible through Taverna, for a very wide range of disparate bioinformatics tasks. However, to the best of our knowledge, the workflow showcased in this work addresses in a sequential, unsupervised fashion disparate tasks enabling and empowering decisively the translational procedure, in a completely innovative yet efficient way.

Applying the proposed workflow to a dataset from ApoE knockout and wild type mice, it was shown that the gene expression profile in atherosclerotic plaques containing arteries of ApoE knockout mice is profoundly different from wild type. Specifically, 852 genes were found as differentially expressed and the majority of them appear after the age of 32 weeks. The indicated altered processes, as revealed by ontology-based enrichment analysis, include adhesion and signal transduction, differentiation, apoptosis, and immune response, reflecting the cellular and molecular complexity of atherosclerosis and the cross-talk of endothelial and immune cells in aortic lesions. Cluster analysis revealed three major groups of genes with similar expression profiles, which were further analyzed, in order to find functional (GO-based) subgroups in each cluster. In agreement with the notion that atherosclerosis is an inflammatory disease [2], immune response and inflammation were the prominent categories indicated as significantly altered in the case of cluster 2 , which contains genes upregulated both in 36- and 78-weeks-old mice. Promoter analysis of the genes under these categories revealed common binding elements that could contribute to a common transcriptional regulation. In particular, all of the tested genes (100\%) contain cis-elements of the KLF and RXR family. The KLF family groups binding sites recognized by Krueppel like transcription factors (KLFs) [28] which are involved in many physiological and pathological processes, such as cell differentiation, proliferation, cell growth, and apoptosis during normal development or under different disease conditions. It is noteworthy that KLFs have been implicated in acute and chronic inflammatory disease states, such as atherosclerosis, diabetes, and airway inflammation [29]. It is important to note that despite the identification of KLF cis-acting elements, Klf4 TF was also found upregulated, suggesting that this factor could be involved in the regulation of the observed stimulation of the immune response related mechanisms. Klf4 has been found to regulate monocyte differentiation and to activate the macrophages to induce inflammation [30]. Furthermore, Klf4 regulates the proliferation and differentiation in vascular smooth muscle cells after injury to the vessel [31]. However, Klf4 seems to have an anti-inflammatory role in endothelial cells [28]. Regarding RXR family, it groups together motifs related to the receptors of retinoids, which are recognized by various heterodimers of retinoid X receptors (RXRs) and retinoic acid receptors. Interestingly, RXR has been reported to 
TABLE 4: GOrevenge prioritization. The second column refers to the number of GO terms remaining after Gorevenge pruning, reflecting the centrality of each gene, while the third column refers to the original number of biological process category GO terms of each gene. Values in the three last columns depict fold changes between ApoE knockout and age-matched wild type mice in log2 scale. All presented genes are also differentially expressed. Top 20 genes are shown.

\begin{tabular}{|c|c|c|c|c|c|}
\hline Gene symbol & Remaining GO terms & Original GO terms & 6 weeks & 32 weeks & 78 weeks \\
\hline Wnt5a & 63 & 112 & 0.04 & -0.38 & -1.9 \\
\hline Fgfr2 & 56 & 92 & 0.15 & -0.39 & -1.05 \\
\hline P2rx7 & 38 & 73 & 0.02 & 0.61 & 1.84 \\
\hline Igf1 & 34 & 56 & -0.23 & 0.77 & 1.39 \\
\hline Thbs1 & 30 & 42 & -0.02 & 1.59 & 1.99 \\
\hline Ptgs2 & 30 & 37 & -0.27 & 1.76 & 1.7 \\
\hline Foxfla & 28 & 34 & 0.09 & -0.63 & -1.41 \\
\hline Psen 2 & 25 & 37 & -0.4 & 0.23 & 1.02 \\
\hline Ccnd1 & 24 & 37 & -0.01 & 0.67 & 1.07 \\
\hline Slc1la1 & 24 & 40 & -0.16 & 1.29 & 1.9 \\
\hline Lyn & 24 & 33 & -0.01 & 1.07 & 2.26 \\
\hline Cebpa & 24 & 30 & -0.3 & 0.21 & 1.77 \\
\hline Tlr2 & 21 & 47 & 0.14 & 0.99 & 1.38 \\
\hline Osr1 & 21 & 33 & 0.09 & 0.08 & -1.41 \\
\hline Hexb & 19 & 23 & -0.04 & 0.67 & 1 \\
\hline Colla1 & 19 & 29 & 0.02 & 0.57 & 1.01 \\
\hline Socs3 & 19 & 27 & 0.58 & 1.59 & 3.19 \\
\hline Adam 17 & 18 & 29 & -0.22 & 0.27 & 1.07 \\
\hline Cd44 & 18 & 20 & 0.15 & 1.46 & 1.63 \\
\hline $\mathrm{Cln} 8$ & 18 & 26 & 0.37 & 0.85 & 1.71 \\
\hline
\end{tabular}

TABle 5: Prioritization of the genes presented in Table 4 by BioGraph exploiting unsupervised methodologies for the identification of causative disease-associated genes.

\begin{tabular}{lc}
\hline Gene symbol & Score \\
\hline PTGS2 & 0.003895 \\
CCND1 & 0.000566 \\
CD44 & 0.000279 \\
COL1A1 & 0.000194 \\
ADAM17 & 0.000168 \\
IGF1 & 0.000116 \\
FGFR2 & 0.000116 \\
THBS1 & 0.000097 \\
LYN & 0.000088 \\
SOCS3 & 0.000087 \\
CEBPA & 0.000054 \\
TLR2 & 0.000048 \\
PSEN2 & 0.000045 \\
P2RX7 & 0.000038 \\
WNT5A & 0.000035 \\
ISLC11A1 & 0.000024 \\
CLN8 & 0.000007 \\
FOXF1 & 0.000006 \\
HEXB & 0.000005 \\
OSR1 & 0.000002 \\
\hline
\end{tabular}

regulate several genes related to metabolic homeostasis and inflammation [32]. RXR form heterodimers with many different nuclear receptors, PPARs, LXR, and FXR affecting different aspects of cholesterol metabolism in macrophages, something known to be important in the development of atherosclerosis [32]. In addition, among the identified putative TF binding sites there are interferon regulatory factorsrelated elements (IRFs) in the $86 \%$ of the promoters, as well as glucocorticoid responsive elements (GREs) in the $92 \%$ of the tested promoters. In agreement, Irf8, a transcription factor involved in modulation of immune response and as a central element in the IFN signaling cascade, was found significantly overexpressed, suggesting that Irf8, together with Klf4, could be involved in the upregulation of the immune response related genes. Regarding GREs, it is well known that glucocorticoid receptors play important roles in both physiological and pathological conditions involving immunity and inflammation and that they are involved in the pathology of cardiovascular diseases [33]. Finally, Table 4 includes several genes implicated to various aspects of the disease. It is noteworthy to mention Tlr2, a member of the Toll-like receptors family, which plays a fundamental role in activation of innate immunity [34]. Furthermore, the identification of Psen2 (presenillin 2), a gene implicated in Alzheimer's disease, as candidate hub gene is interesting because genes implicated in Alzheimer's have been reported to affect cholesterol or lipoprotein function and have also been implicated in atherosclerosis [35].

Concluding, this bioinformatic analysis of ApoE knockout mice revealed critical altered cellular mechanisms governing atherosclerosis and indicated important molecular players that could be important targets for treatment of this complex disease. 


\section{References}

[1] G. Stoll and M. Bendszus, "Inflammation and atherosclerosis: novel insights into plaque formation and destabilization," Stroke, vol. 37, no. 7, pp. 1923-1932, 2006.

[2] G. K. Hansson, "Mechanisms of disease: inflammation, atherosclerosis, and coronary artery disease," The New England Journal of Medicine, vol. 352, no. 16, pp. 1685-1695, 2005.

[3] S. H. Zhang, R. L. Reddick, J. A. Piedrahita, and N. Maeda, "Spontaneous hypercholesterolemia and arterial lesions in mice lacking apolipoprotein E," Science, vol. 258, no. 5081, pp. 468-471, 1992.

[4] R. W. Mahley and S. C. Rall Jr., "Apolipoprotein E: far more than a lipid transport protein," Annual Review of Genomics and Human Genetics, vol. 1, no. 2000, pp. 507-537, 2000.

[5] Y. Song, M. J. Stampfer, and S. Liu, "Meta-analysis: apolipoprotein E genotypes and risk for coronary heart disease," Annals of Internal Medicine, vol. 141, no. 2, pp. 137 147, 2004.

[6] E. Newcomer and G. Lomow, Understanding SOA with Web Services, Addison-Wesley, New York, NY, USA, 2004.

[7] R. C. Gentleman, V. J. Carey, D. M. Bates et al., "Bioconductor: open software development for computational biology and bioinformatics," Genome Biology, vol. 5, no. 10, article R80, 2004.

[8] A. Chatziioannou, P. Moulos, and F. N. Kolisis, "Gene ARMADA: an integrated multi-analysis platform for microarray data implemented in MATLAB," BMC Bioinformatics, vol. 10, article 354, 2009.

[9] M. M. Michal Blazejczyk and R. Nadon, "FlexArray: a statistical data analysis software for gene expression microarrays," Génome Québec, Montreal, Canada, 2007, http://genomequebec.mcgill.ca/FlexArray.

[10] G. Dennis Jr., B. T. Sherman, D. A. Hosack et al., "DAVID: database for annotation, visualization, and integrated discovery," Genome Biology, vol. 4, no. 5, article P3, 2003.

[11] D. A. Hosack, G. Dennis Jr., B. T. Sherman, H. C. Lane, and R. A. Lempicki, "Identifying biological themes within lists of genes with EASE," Genome Biology, vol. 4, no. 10, article R70, 2003.

[12] B. Zhang, S. Kirov, and J. Snoddy, "WebGestalt: an integrated system for exploring gene sets in various biological contexts," Nucleic Acids Research, vol. 33, no. 2, pp. W741-W748, 2005.

[13] Q. Zheng and X. J. Wang, "GOEAST: a web-based software toolkit for gene ontology enrichment analysis," Nucleic Acids Research, vol. 36, pp. W358-W363, 2008.

[14] A. A. Chatziioannou and P. Moulos, "Exploiting statistical methodologies and controlled vocabularies for prioritized functional analysis of genomic experiments: the StRAnGER web application," Frontiers in Neuroscience, vol. 5, article 8.

[15] Genomatix, http://www.genomatix.de.

[16] Z. Xuan, F. Zhao, J. Wang, G. Chen, and M. Q. Zhang, "Genome-wide promoter extraction and analysis in human, mouse, and rat," Genome Biology, vol. 6, no. 8, article R72, 2005.

[17] A. Essaghir, F. Toffalini, L. Knoops, A. Kallin, J. van Helden, and J. B. Demoulin, "Transcription factor regulation can be accurately predicted from the presence of target gene signatures in microarray gene expression data," Nucleic Acids Research, vol. 38, no. 11, article e120, 2010.

[18] K. Cartharius, K. Frech, K. Grote et al., "MatInspector and beyond: promoter analysis based on transcription factor binding sites," Bioinformatics, vol. 21, no. 13, pp. 2933-2942, 2005.
[19] C. Pesquita, D. Faria, A. O. Falcão, P. Lord, and F. M. Couto, "Semantic similarity in biomedical ontologies," PLoS Computational Biology, vol. 5, no. 7, Article ID e1000443, 2009.

[20] K. Moutselos, I. Maglogiannis, and A. Chatziioannou, "GOrevenge: a novel generic reverse engineering method for the identification of critical molecular players, through the use of ontologies," IEEE Transactions on Biomedical Engineering, vol. 58, no. 12, pp. 3522-3527, 2011.

[21] A. M. L. Liekens, J. de Knijf, W. Daelemans, B. Goethals, P. de Rijk, and J. Del-Favero, "Biograph: unsupervised biomedical knowledge discovery via automated hypothesis generation," Genome Biology, vol. 12, no. 6, article R57, 2011.

[22] R. Gräbner, K. Lötzer, S. Döpping et al., "Lymphotoxin $\beta$ receptor signaling promotes tertiary lymphoid organogenesis in the aorta adventitia of aged ApoE-/- mice," Journal of Experimental Medicine, vol. 206, no. 1, pp. 233-248, 2009.

[23] HomoloGene, http://www.ncbi.nlm.nih.gov/homologene.

[24] T. Oinn, M. Addis, J. Ferris et al., "Taverna: a tool for the composition and enactment of bioinformatics workflows," Bioinformatics, vol. 20, no. 17, pp. 3045-3054, 2004.

[25] I. Mierswa, M. Scholz, R. Klinkenberg, M. Wurst, and T. Euler, "YALE: rapid prototyping for complex data mining tasks," in Proceedings of the 12th ACM SIGKDD International Conference on Knowledge Discovery and Data Mining, pp. 935-940, August 2006.

[26] C. A. Goble, J. Bhagat, S. Aleksejevs et al., "myExperiment: a repository and social network for the sharing of bioinformatics workflows," Nucleic Acids Research, vol. 38, no. 2, pp. W677-W682, 2010.

[27] J. Bhagat, F. Tanoh, E. Nzuobontane et al., "BioCatalogue: a universal catalogue of web services for the life sciences," Nucleic Acids Research, vol. 38, no. 2, pp. W689-W694, 2010.

[28] A. Hamik, Z. Lin, A. Kumar et al., "Kruppel-like factor 4 regulates endothelial inflammation," The Journal of Biological Chemistry, vol. 282, no. 18, pp. 13769-13779, 2007.

[29] Z. Cao, X. Sun, B. Icli, A. K. Wara, and M. W. Feinberg, "Role of Krüppel-like factors in leukocyte development, function, and disease," Blood, vol. 116, no. 22, pp. 4404-4414, 2010.

[30] J. K. Alder, R. W. Georgantas, R. L. Hildreth et al., "Kruppellike factor 4 is essential for inflammatory monocyte differentiation in vivo," Journal of Immunology, vol. 180, no. 8, pp. 5645$5652,2008$.

[31] T. Yoshida, Q. Gan, and G. K. Owens, "Krüppel-like factor 4, Elk-1, and histone deacetylases cooperatively suppress smooth muscle cell differentiation markers in response to oxidized phospholipids," American Journal of Physiology, vol. 295, no. 5, pp. C1175-C1182, 2008.

[32] A. Nohara, J. Kobayashi, and H. Mabuchi, "Retinoid X receptor heterodimer variants and cardiovascular risk factors," Journal of Atherosclerosis and Thrombosis, vol. 16, no. 4, pp. 303-318, 2009.

[33] P. W. F. Hadoke, J. Iqbal, and B. R. Walker, "Therapeutic manipulation of glucocorticoid metabolism in cardiovascular disease," British Journal of Pharmacology, vol. 156, no. 5, pp. 689-712, 2009.

[34] S. Borrello, C. Nicolo, G. Delogu, F. Pandolfi, and F. Ria, "TLR2: a crossroads between infections and autoimmunity?" International Journal of Immunopathology and Pharmacology, vol. 24, no. 3, pp. 549-556.

[35] C. J. Carter, "Convergence of genes implicated in Alzheimer's disease on the cerebral cholesterol shuttle: APP, cholesterol, lipoproteins, and atherosclerosis," Neurochemistry International, vol. 50, no. 1, pp. 12-38, 2007. 

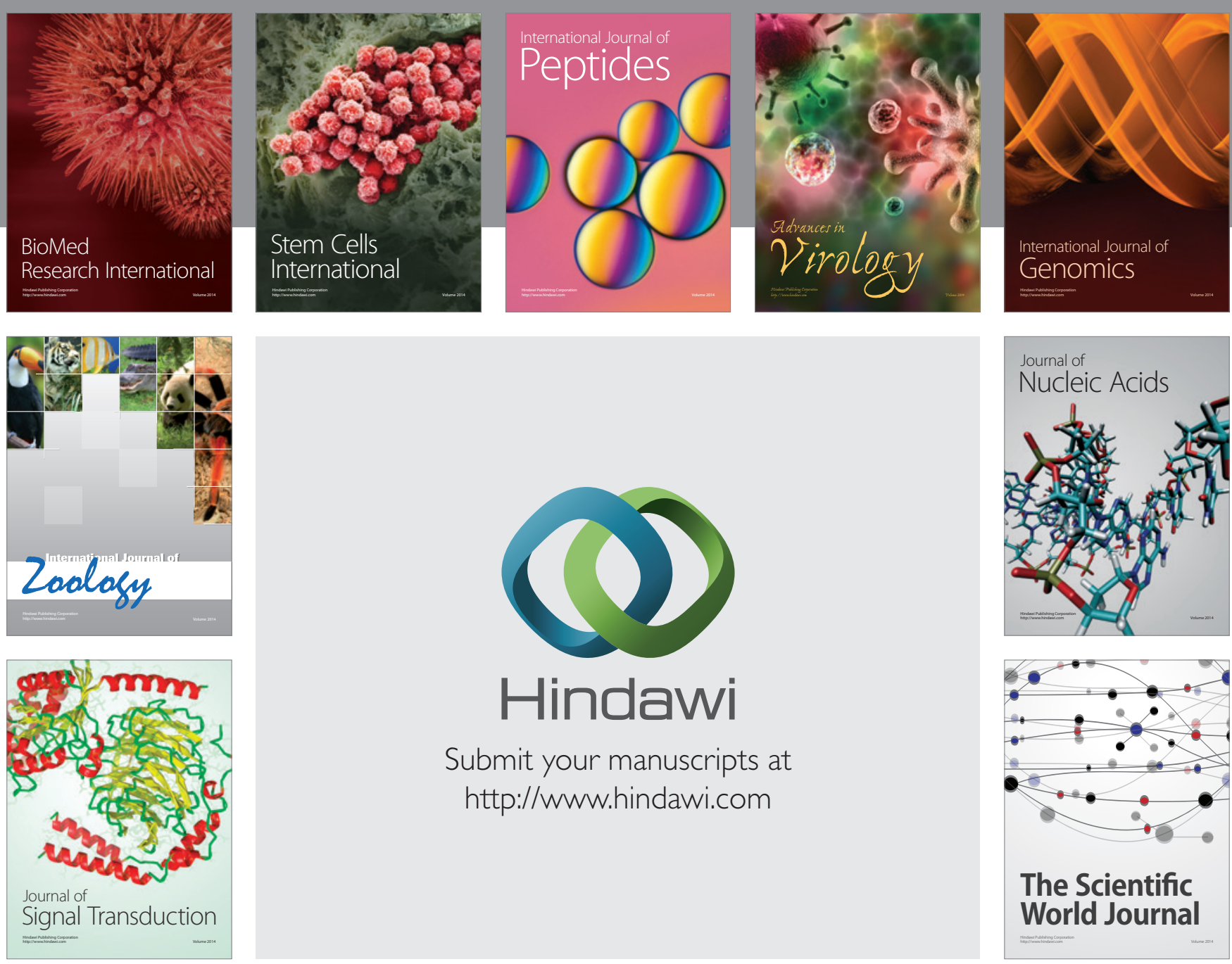

Submit your manuscripts at

http://www.hindawi.com
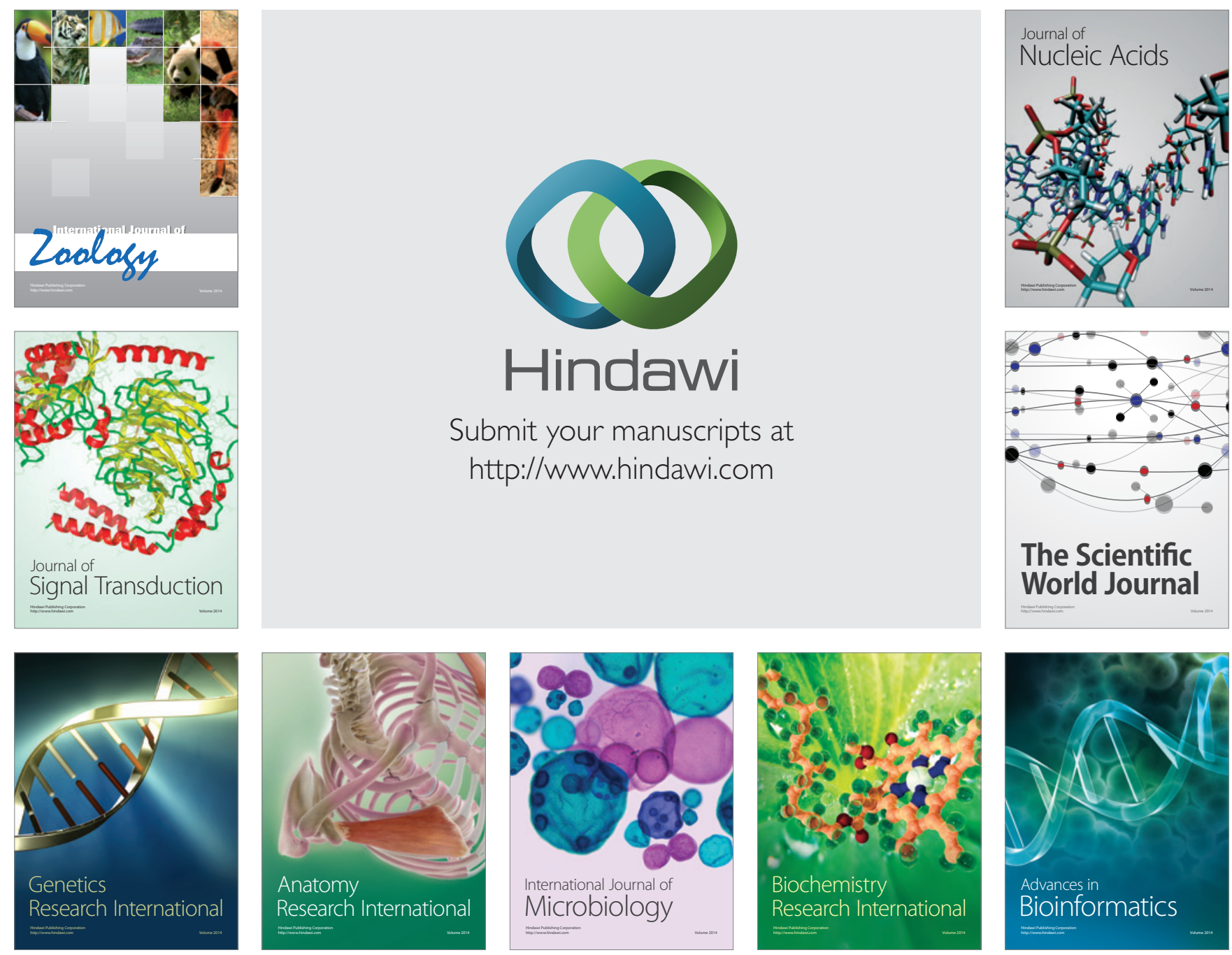

The Scientific World Journal
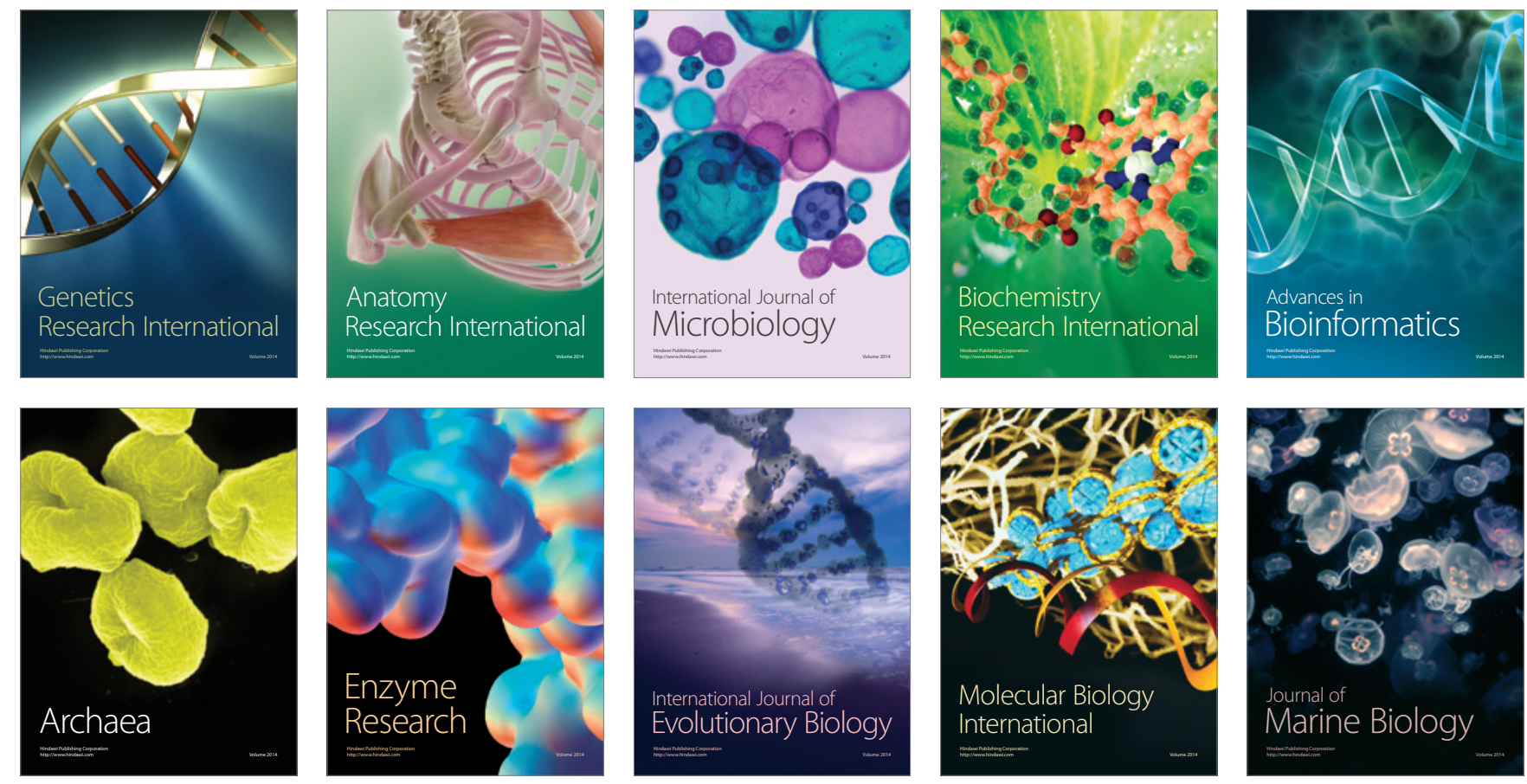